\title{
Lower bounds on concurrence and separability conditions
}

\author{
Julio I. de Vicente \\ Departamento de Matemáticas, Universidad Carlos III de Madrid, \\ Avda. de la Universidad 30, 28911 Leganés, Madrid, Spain
}

\begin{abstract}
We obtain analytical lower bounds on the concurrence of bipartite quantum systems in arbitrary dimensions related to the violation of separability conditions based on local uncertainty relations and on the Bloch representation of density matrices. We also illustrate how these results complement and improve those recently derived [K. Chen, S. Albeverio, and S.-M. Fei, Phys. Rev. Lett. 95, 040504 (2005)] by considering the Peres-Horodecki and the computable cross norm or realignment criteria.
\end{abstract}

PACS numbers: 03.67.Mn, 03.65.Ud

\section{INTRODUCTION}

Entanglement is an essential ingredient in many applications of quantum information theory such as dense coding, teleportation, quantum cryptography and quantum computing [1]. Therefore, the characterization and quantification of entanglement are of great importance in this field. However, and despite many efforts in the last decade, a completely satisfactory solution to both problems has not been found. Attending to the first one (the so-called separability problem), there exist, nevertheless, several sufficient conditions for the detection of entanglement. The most powerful is known as the PeresHorodecki or positive partial transpose (PPT) criterion [2], which is also necessary for low-dimensional systems $(2 \times 2$ and $2 \times 3)[3]$. Another remarkable sufficient condition is given by the computable cross norm [4] or realignment 5] (CCNR) criterion, which allows to detect many entangled states for which the PPT criterion fails. Recently, another criterion with this property [6] has been developed by the author which we shall denote as correlation matrix (CM) criterion. There are also other important criteria, which, however, lack the operational character of the aforementioned ones, since they are stated in terms of mean values or variances of observables which have to be chosen wisely. This is the case of conditions based on entanglement witnesses (EWs) 3, 7] or uncertainty relations [8, 9]. In what comes to the quantification of entanglement, there exist a large variety of proposed measures [10]. However, the explicit computation of these measures for arbitrary states is a very hard task, not only analytically but also from the computational point of view since they require optimization over a large number of parameters [31]. The only measures for which an analytical expression is available is the entanglement of formation [11] and the concurrence [12, 13], for which Wootters [12] derived a formula in the case of two qubits. Given the aforementioned difficulties for the evaluation of the concurrence for higher dimensions, good bounds for the estimation of this quantity have been sought. While, by construction, upper bounds are numerically affordable, the derivation of lower bounds has demanded a more thorough analysis [14]. A completely analyti- cal and powerful lower bound for the concurrence was found in [15] by relating this quantity with the PPT and CCNR criteria, giving shape, therefore, to the intuitive idea that a stronger violation of a separability condition may indicate a higher amount of entanglement. In fact, a possible connection between the value of concurrence and the violation of a separability condition based on local uncertainty relations (LURs) was already suggested in [8] (see [16, 17] for further discussions on LURs and the quantification of entanglement). The aim of this paper is to sharpen the bounds of [15] by relating concurrence and the LURs and CM criteria, giving as a by-product a deeper insight in the above idea. Partial improvements on these bounds have already been achieved in the particular case of $N \times N$ quantum systems with even $N \geq 4$ in 18] by considering an EW based separability criterion [19], but the approach here is valid for the general case. The bounds imposed by measurements of arbitrary EWs on different entanglement measures have been recently studied in [20].

\section{NEW LOWER BOUNDS ON CONCURRENCE}

We start by recalling the definitions of the concepts and quantities we are dealing with. Let $H_{A} \simeq \mathbb{C}^{M}$ and $H_{B} \simeq \mathbb{C}^{N}$ denote the Hilbert spaces of subsystems $A$ and $B(M \leq N)$. Then, the quantum state of the total system is characterized by the density operator $\rho \in \mathcal{B}\left(H_{A} \otimes H_{B}\right)$, where $\mathcal{B}(H)$ stands for the real vector space of Hermitian operators acting on $H$, which is a Hilbert-Schmidt space (with inner product $\langle\rho, \tau\rangle_{H S}=\operatorname{Tr}\left(\rho^{\dagger} \tau\right)$ ). The state is said to be separable (entangled) if it can (cannot) be written as a convex combination of product states [21], i. e. $\rho=\sum_{i} p_{i} \rho_{i}^{A} \otimes \rho_{i}^{B}$ where $0 \leq p_{i} \leq 1, \sum_{i} p_{i}=1$, and $\rho_{i}^{A}\left(\rho_{i}^{B}\right)$ denotes a pure state density matrix acting on $H_{A}\left(H_{B}\right)$. The generalized definition [13] for the concurrence of a pure state $\psi$ is given by $C(\psi)=\sqrt{2\left(1-\operatorname{Tr} \rho_{A}^{2}\right)}$, where the reduced density matrix $\rho_{A}$ is obtained by tracing out subsystem $B\left(\rho_{A}=\operatorname{Tr}_{B}|\psi\rangle\langle\psi|\right)$. Notice that $0 \leq C(\psi) \leq \sqrt{2(M-1) / M}$, the lower bound being attained by product states and the upper bound by max- 
imally entangled states. The definition is extended to general mixed states $\rho$ by the convex roof,

$$
C(\rho)=\min _{\left\{p_{i},\left|\psi_{i}\right\rangle\right\}}\left\{\sum_{i} p_{i} C\left(\psi_{i}\right): \rho=\sum_{i} p_{i}\left|\psi_{i}\right\rangle\left\langle\psi_{i}\right|\right\} .
$$

Consequently, $C(\rho)=0$ if, and only if, $\rho$ is a separable state. The PPT and CCNR criteria can be formulated in several ways. Basically, they state that certain rearrangements of the matrix elements of $\rho$ [22], namely, the partial transpose $T_{A}(\rho)$ (PPT criterion) and the realignment $R(\rho)$ (CCNR criterion), are such that for separable states $\left\|T_{A}(\rho)\right\|=1$ and $\|R(\rho)\| \leq 1$, where here, and throughout the paper, $\|\cdot\|$ stands for the trace norm (i.e. the sum of the singular values). By directly relating both criteria and concurrence by means of the Schmidt coefficients of a pure state it was found in [15] that

$$
C(\rho) \geq \sqrt{\frac{2}{M(M-1)}}\left[\max \left(\left\|T_{A}(\rho)\right\|,\|R(\rho)\|\right)-1\right] .
$$

\section{A. LURs criterion}

One of the most interesting separability criteria based on uncertainty relations is that of LURs 8], since it can detect PPT entanglement [23, 24]. It states that if $\left\{A_{i}\right\}$ and $\left\{B_{i}\right\}$ are observables acting on $H_{A}$ and $H_{B}$ respectively, fulfilling uncertainty relations $\sum_{i} \Delta_{\rho}^{2}\left(A_{i}\right) \geq C_{A}$ and $\sum_{i} \Delta_{\rho}^{2}\left(B_{i}\right) \geq C_{B}\left(C_{A}, C_{B} \geq 0\right)$, then,

$$
\sum_{i} \Delta_{\rho}^{2}\left(A_{i} \otimes I+I \otimes B_{i}\right) \geq C_{A}+C_{B}
$$

holds for separable states [8]. The variance $\Delta^{2}$ is given by $\Delta_{\rho}^{2}(M)=\left\langle M^{2}\right\rangle_{\rho}-\langle M\rangle_{\rho}^{2}$, where $\langle M\rangle_{\rho}=\operatorname{Tr}(\rho M)$ is the expectation value of the observable $M$. A particularly interesting choice for the observables is that of local orthogonal observables (LOOs) 25], that is, orthonormal bases of $\mathcal{B}\left(H_{A}\right)$ and $\mathcal{B}\left(H_{B}\right)$, which we shall denote $\left\{G_{i}^{A}\right\}_{i=1}^{M^{2}}$ and $\left\{G_{i}^{B}\right\}_{i=1}^{N^{2}}$. In this case Eq. (3) reads 24]

$$
\sum_{i=1}^{N^{2}} \Delta_{\rho}^{2}\left(G_{i}^{A} \otimes I+I \otimes G_{i}^{B}\right) \geq M+N-2
$$

since

$$
\sum_{i=1}^{M^{2}} \Delta_{\rho}^{2}\left(G_{i}^{A}\right) \geq M-1, \quad \sum_{i=1}^{N^{2}} \Delta_{\rho}^{2}\left(G_{i}^{B}\right) \geq N-1 .
$$

Notice that if $M<N$, in Eq. (4) (and throughout the paper) it is understood that $G_{i}^{A}=0$ for $M^{2}+1 \leq i \leq$ $N^{2}$. The standard set of LOOs is given by $\left\{G_{i}^{A}\right\}=$ $\left\{g_{j}, g_{j k}^{+}, g_{j k}^{-}\right\}$where

$$
\begin{aligned}
g_{j} & =|j\rangle\langle j| \quad(0 \leq j \leq M-1), \\
g_{j k}^{+} & =\frac{1}{\sqrt{2}}(|j\rangle\langle k|+| k\rangle\langle j|) \quad(0 \leq j<k \leq M-1), \\
g_{j k}^{-} & =-\frac{i}{\sqrt{2}}(|j\rangle\langle k|-| k\rangle\langle j|) \quad(0 \leq j<k \leq M-1),
\end{aligned}
$$

and similarly for $\left\{G_{i}^{B}\right\}$. The importance of the LURs condition formulated in terms of LOOs relies on that it is strictly stronger than the CCNR condition 24]. Furthermore, it can detect entangled states for which both the PPT and CCNR criteria fail 24]. In order to relate concurrence and LURs with LOOs analogously as in Eq. (2) we start with the following lemma:

Lemma 1 For any set of LOOs $\left\{G_{i}^{A}\right\}$ and $\left\{G_{i}^{B}\right\}$ and any $M \times N(M \leq N)$ pure state $\psi$ with Schmidt decomposition $|\psi\rangle=\sum_{j=0}^{M-1} \sqrt{\mu_{j}}\left|j_{A} j_{B}\right\rangle$,

$$
\sum_{i=1}^{N^{2}} \Delta_{\psi}^{2}\left(G_{i}^{A} \otimes I+I \otimes G_{i}^{B}\right) \geq M+N-2-4 \sum_{j<k} \sqrt{\mu_{j} \mu_{k}}
$$

holds. The bound is attained when $\left\{G_{i}^{A}\right\}=\left\{g_{j}, g_{j k}^{+}, g_{j k}^{-}\right\}$ and $\left\{G_{i}^{B}\right\}=\left\{-g_{j},-g_{j k}^{+}, g_{j k}^{-}\right\}$(constructed from the corresponding Schmidt basis).

Proof. We have that

$$
\begin{aligned}
\sum_{i} \Delta_{\psi}^{2}\left(G_{i}^{A} \otimes I+I \otimes G_{i}^{B}\right) & =\sum_{i}\left(\Delta_{\rho_{A}}^{2}\left(G_{i}^{A}\right)+\Delta_{\rho_{B}}^{2}\left(G_{i}^{B}\right)\right) \\
& +2 \sum_{i} \kappa_{\psi}\left(G_{i}^{A}, G_{i}^{B}\right),
\end{aligned}
$$

where

$$
\kappa_{\psi}\left(G_{i}^{A}, G_{i}^{B}\right)=\left\langle G_{i}^{A} \otimes G_{i}^{B}\right\rangle_{\psi}-2\left\langle G_{i}^{A}\right\rangle_{\rho_{A}}\left\langle G_{i}^{B}\right\rangle_{\rho_{B}} .
$$

Let us write $\rho_{\psi}=|\psi\rangle\langle\psi|=\rho^{\text {sep }}+\epsilon$, where $\rho^{\text {sep }}=$ $\sum_{j} \mu_{j}\left|j_{A} j_{B}\right\rangle\left\langle j_{A} j_{B}\right|$ and $\epsilon=\sum_{j \neq k} \sqrt{\mu_{j} \mu_{k}}\left|j_{A} j_{B}\right\rangle\left\langle k_{A} k_{B}\right|$. Notice that $\rho^{\text {sep }}$ is a separable state and that its reductions are the same as those of $\rho_{\psi}\left(\rho_{A}^{s e p}=\rho_{A}, \rho_{B}^{s e p}=\rho_{B}\right)$. Thus,

$$
\kappa_{\psi}\left(G_{i}^{A}, G_{i}^{B}\right)=\operatorname{Tr}\left(\epsilon G_{i}^{A} \otimes G_{i}^{B}\right)+\kappa_{\rho^{s e p}}\left(G_{i}^{A}, G_{i}^{B}\right) .
$$

Now, since LURs hold for separable states we have that

$$
\begin{aligned}
2 \sum_{i} \kappa_{\rho^{s e p}}\left(G_{i}^{A}, G_{i}^{B}\right) & \geq M+N-2 \\
& -\sum_{i}\left(\Delta_{\rho_{A}}^{2}\left(G_{i}^{A}\right)+\Delta_{\rho_{B}}^{2}\left(G_{i}^{B}\right)\right)
\end{aligned}
$$

and then,

$\sum_{i} \Delta_{\psi}^{2}\left(G_{i}^{A} \otimes I_{B}+I_{A} \otimes G_{i}^{B}\right) \geq M+N-2+2 \sum_{i} \operatorname{Tr}\left(\epsilon G_{i}^{A} \otimes G_{i}^{B}\right)$, 
so that it remains to prove that $X \equiv \sum_{i} \operatorname{Tr}\left(\epsilon G_{i}^{A} \otimes G_{i}^{B}\right) \geq$ $-2 \sum_{j<k} \sqrt{\mu_{j} \mu_{k}}$. To do so, notice that

$$
\begin{aligned}
X & =\sum_{i} \sum_{j \neq k} \sqrt{\mu_{j} \mu_{k}}\left\langle j_{A}\left|G_{i}^{A}\right| k_{A}\right\rangle\left\langle j_{B}\left|G_{i}^{B}\right| k_{B}\right\rangle \\
& \geq-\sum_{i} \sum_{j \neq k} \sqrt{\mu_{j} \mu_{k}}\left|\left\langle j_{A}\left|G_{i}^{A}\right| k_{A}\right\rangle\left\langle j_{B}\left|G_{i}^{B}\right| k_{B}\right\rangle\right| \\
& \geq-\sum_{i} \sum_{j \neq k} \sqrt{\mu_{j} \mu_{k}}\left(\left|\left\langle j_{A}\left|G_{i}^{A}\right| k_{A}\right\rangle\right|^{2}+\left|\left\langle j_{B}\left|G_{i}^{B}\right| k_{B}\right\rangle\right|^{2}\right) / 2,
\end{aligned}
$$

where in the last step we have used that $a^{2}+b^{2} \geq 2|a b|$. Now, the result follows because $\sum_{i}\left|\left\langle j_{A}\left|G_{i}^{A}\right| k_{A}\right\rangle\right|^{2}=$ $\sum_{i}\left|\left\langle j_{B}\left|G_{i}^{B}\right| k_{B}\right\rangle\right|^{2}=1 \forall j, k$ for any set of LOOs $\left\{G_{i}^{A}\right\}$ and $\left\{G_{i}^{B}\right\}$. To see this, consider that $\mathcal{B}\left(H_{A}\right)$ is isomorphic to $\mathbb{C}^{M^{2}}$ with the standard inner product, so that the $\left\{G_{i}^{A}\right\}$ can be arranged as column vectors which give an orthonormal basis of this space. This column vectors together give rise to a unitary matrix $U$, and $\sum_{i}\left|\left\langle j_{A}\left|G_{i}^{A}\right| k_{A}\right\rangle\right|^{2}$ corresponds to summing the squared modulus of the elements of a certain row of $U$ and, therefore, it equals unity. Obviously the same reasoning holds for $\sum_{i}\left|\left\langle j_{B}\left|G_{i}^{B}\right| k_{B}\right\rangle\right|^{2}$. It remains to check that the bound is attained by the above stated set of LOOs. Using that $\sum_{i}\left(G_{i}^{A}\right)^{2}=M I$ and $\sum_{i}\left(G_{i}^{B}\right)^{2}=N I$ [24], it is straightforward to find that

$$
\begin{aligned}
\sum_{i=1}^{N^{2}} \Delta_{\psi}^{2}\left(G_{i}^{A} \otimes I+I \otimes G_{i}^{B}\right) & =M+N+2 \sum_{i}\left\langle G_{i}^{A} \otimes G_{i}^{B}\right\rangle_{\psi} \\
& -\sum_{i}\left\langle G_{i}^{A} \otimes I+I \otimes G_{i}^{B}\right\rangle_{\psi}^{2} .
\end{aligned}
$$

Considering that any pure density matrix $\rho_{\psi}$ achieves its Schmidt decomposition for the standard LOOs, i.e. $\rho_{\psi}=\sum_{j} \mu_{j} g_{j} \otimes g_{j}+\sum_{j<k} \sqrt{\mu_{j} \mu_{k}}\left(g_{j k}^{+} \otimes g_{j k}^{+}+g_{j k}^{-} \otimes\left(-g_{j k}^{-}\right)\right)$, it follows that $\sum_{i}\left\langle G_{i}^{A} \otimes G_{i}^{B}\right\rangle_{\psi}=-1-2 \sum_{j<k} \sqrt{\mu_{j} \mu_{k}}$ and that $\left\langle G_{i}^{A} \otimes I+I \otimes G_{i}^{B}\right\rangle_{\psi}=0 \forall i$ for the set of LOOs mentioned in the statement of the lemma, and the result is thus proved.

Now, we can prove our main result.

Theorem 1 For any $M \times N(M \leq N)$ quantum state $\rho$,

$$
C(\rho) \geq \frac{M+N-2-\sum_{i} \Delta_{\rho}^{2}\left(G_{i}^{A} \otimes I+I \otimes G_{i}^{B}\right)}{\sqrt{2 M(M-1)}}
$$

holds for any set of LOOs $\left\{G_{i}^{A}\right\}$ and $\left\{G_{i}^{B}\right\}$.

Proof. Let $\sum_{n} p_{n}\left|\psi_{n}\right\rangle\left\langle\psi_{n}\right|$ be the decomposition of $\rho$ for which the minimum in Eq. (11) is attained, so that, $C(\rho)=\sum_{n} p_{n} C\left(\psi_{n}\right)$. Since the concurrence of a pure state is directly related to its Schmidt coefficients [13]: $C^{2}\left(\psi_{n}\right)=4 \sum_{j<k} \mu_{j} \mu_{k}$, and 15]

$$
\sum_{j<k} \mu_{j} \mu_{k} \geq \frac{2}{M(M-1)}\left(\sum_{j<k} \sqrt{\mu_{j} \mu_{k}}\right)^{2},
$$

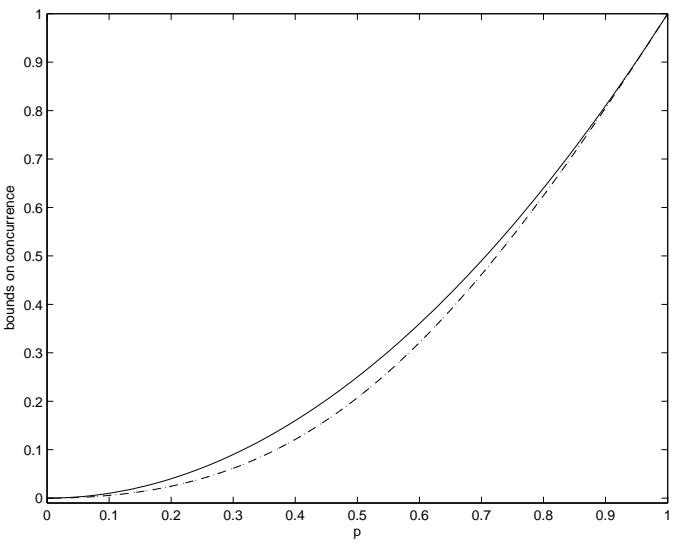

Figure 1: Lower bounds on concurrence for the the state $\varrho$. Solid line: Bound given by the LURs criterion with the LOOs that achieve the bound in Eq. (7) for the state $\Psi$. Dotted line: Bound given by the PPT criterion. Dashed line: Bound given by the CCNR criterion. (The last two bounds overlap).

we have that $C\left(\psi_{n}\right) \geq \sqrt{2 /(M(M-1))} 2 \sum_{j<k} \sqrt{\mu_{j} \mu_{k}}$. Now, the use of Lemma 1 and the fact that $\Delta_{\rho}^{2}(M) \geq$ $\sum_{n} p_{n} \Delta_{\psi_{n}}^{2}(M)$ for any observable $M$ (see e.g. [8]) proves the desired result.

Next we present a couple of examples which illustrate how this result can improve on the bounds of [15]. First, consider the case of PPT entangled states. In this case any non-trivial bound on concurrence given by Eq. (2) must rely on the CCNR criterion. However, the LURs criterion can identify states of this kind which are not detected by the CCNR criterion and, therefore, place a non-trivial bound on concurrence where the previous approach failed (see [24]). Furthermore, Eq. (8) can improve the estimation of PPT entanglement of Eq. (2) even when the latter supplies a non-trivial bound. For instance, consider the following $3 \times 3 \mathrm{PPT}$ entangled state constructed in [26] from unextendible product bases (UPB): $\rho=1 / 4\left(I-\sum_{i}\left|\psi_{i}\right\rangle\left\langle\psi_{i}\right|\right)$, where $\left|\psi_{0}\right\rangle=|0\rangle(|0\rangle-|1\rangle) / \sqrt{2},\left|\psi_{1}\right\rangle=(|0\rangle-|1\rangle)|2\rangle / \sqrt{2}$, $\left|\psi_{2}\right\rangle=|2\rangle(|1\rangle-|2\rangle) / \sqrt{2},\left|\psi_{3}\right\rangle=(|1\rangle-|2\rangle)|0\rangle / \sqrt{2}$ and $\left|\psi_{4}\right\rangle=(|0\rangle+|1\rangle+|2\rangle)(|0\rangle+|1\rangle+|2\rangle) / 3$. While Eq. (2) yields $C(\rho) \geq 0.050[15]$, Theorem 1 with the LOOs used in 24] to improve the detection of $\rho$ mixed with white noise gives $C(\rho) \geq 0.052$. Another interesting example is to consider the $2 \times 3$ state $\varrho=p|\Psi\rangle\langle\Psi|+(1-p)| 01\rangle\langle 01|$, where $|\Psi\rangle=(|00\rangle+|11\rangle) / \sqrt{2}$, which shows that (8) can give a better bound for the concurrence than (2) even though the PPT criterion characterizes entanglement optimally in this case (see Fig. 1).

\section{B. CM criterion}

Besides its PPT entanglement detection capability, the CM criterion can be stronger than the CCNR criterion when $M \neq N[\underline{6}]$, so it seems worthwhile to derive an 
analogous result relying on this criterion. It is based on the Bloch representation of density matrices which is achieved by expanding $\rho$ with respect to a particular set of unnormalized LOOs, namely, the identity and the traceless Hermitian generators of $S U(M)$ and $S U(N)$ (denoted $\left\{\lambda_{i}^{A}\right\}_{i=1}^{M^{2}-1}$ and $\left\{\lambda_{i}^{B}\right\}_{i=1}^{N^{2}-1}$ hereafter), i.e. $\rho=1 / M N\left(I \otimes I+\sum_{i} r_{i} \lambda_{i}^{A} \otimes I+\sum_{j} s_{j} I \otimes \lambda_{j}^{B}+\right.$ $\left.\sum_{i, j} t_{i j} \lambda_{i}^{A} \otimes \lambda_{j}^{B}\right)$. The generators $\left\{\lambda_{i}^{A}\right\}=\left\{w_{l}, u_{j k}, v_{j k}\right\}$ of $S U(M)$ can be constructed from any orthonormal basis in $H_{A}$ [27,

$$
\begin{aligned}
& w_{l}=\sqrt{\frac{2}{(l+1)(l+2)}}\left(\sum_{i=0}^{l}|i\rangle\langle i|-(l+1)| l+1\rangle\langle l+1|\right), \\
& u_{j k}=\sqrt{2} g_{j k}^{+}, \quad v_{j k}=\sqrt{2} g_{j k}^{-},
\end{aligned}
$$

where $0 \leq l \leq M-2$ and $0 \leq j<k \leq M-1$. The Bloch representation has two kind of parameters: $\left\{r_{i}\right\}$ and $\left\{s_{i}\right\}$, which are local since they are the Bloch parameters of the reductions $\left(\rho_{A}=1 / M\left(I+\sum_{i} r_{i} \lambda_{i}^{A}\right), \rho_{B}=\right.$ $\left.1 / N\left(I+\sum_{i} s_{i} \lambda_{i}^{B}\right)\right) ;$ and $\left\{t_{i j}\right\}=M N / 4\left\{\left\langle\lambda_{i}^{A} \otimes \lambda_{j}^{B}\right\rangle_{\rho}\right\}$, which are responsible for the possible correlations between the subsystems. These last coefficients can be arranged to form the CM, $(T)_{i j}=t_{i j}$. The CM criterion affirms that there is an upper bound to the correlations inherent in a separable state since $\|T\| \leq K_{M N}=$ $\sqrt{M N(M-1)(N-1)} / 2$ must hold for these states [6].

Theorem 2 For any $M \times N(M \leq N)$ quantum state $\rho$,

$$
C(\rho) \geq \sqrt{\frac{8}{M^{3} N^{2}(M-1)}}\left(\|T\|-K_{M N}\right)
$$

holds.

Proof. As before, let us first relate $\left\|T_{\psi}\right\|$ of a pure state to its Schmidt coefficients. Following the notation of the proof of Lemma 1 we write the pure state density matrix as $\rho_{\psi}=\rho^{s e p}+\epsilon=\rho^{s e p}+1 / 2 \sum_{j<k} \sqrt{\mu_{j} \mu_{k}}\left(u_{j k} \otimes u_{j k}-\right.$ $\left.v_{j k} \otimes v_{j k}\right)$. Since $\rho^{s e p}$ is diagonal, its Bloch representation is just given in terms of the identity and the $w_{l}$ 's. Therefore, the CM of $\rho_{\psi}$ is block-diagonal and, thus, $\left\|T_{\psi}\right\|=$ $\left\|T_{\rho^{s e p}}\right\|+M N \sum_{j<k} \sqrt{\mu_{j} \mu_{k}} \leq K_{M N}+M N \sum_{j<k} \sqrt{\mu_{j} \mu_{k}}$. Hence, using again Eq. (9) we have that

$$
C(\psi) \geq \sqrt{\frac{8}{M^{3} N^{2}(M-1)}}\left(\left\|T_{\psi}\right\|-K_{M N}\right) .
$$

Let $\sum_{n} p_{n}\left|\psi_{n}\right\rangle\left\langle\psi_{n}\right|$ denote the ensemble decomposition of $\rho$ for which $C(\rho)=\sum_{n} p_{n} C\left(\psi_{n}\right)$. Then, we can use the above inequality for every $\psi_{n}$ together with the triangle inequality $\left(\left\|T_{\rho}\right\|=\left\|\sum_{n} p_{n} T_{\psi_{n}}\right\| \leq \sum_{n} p_{n}\left\|T_{\psi_{n}}\right\|\right)$ to prove the claim.

Regrettably, to find examples in which Eq. (11) improves the bound given by Eq. (2) is harder than in the case of LURs. This is, among other reasons, because the norm of the CM of a pure state is related to the Schmidt coefficients through an inequality, while in the
PPT and CCNR cases this kind of relation was given by equality. Thus, Theorem 2 is only expected to improve on the result of [15] for states which are detected by the CM criterion but not by the PPT and CCNR criteria, or, more generally, in situations where the former criterion is stronger than both the later criteria at the same time [29].

\section{CONCLUSIONS}

We have derived an analytical lower bound for the concurrence related to the LURs criterion for separability. We have shown by considering explicit examples how this result can improve the bounds given in [15], which rely on the PPT and CCNR criteria. We have also shown that this new result can yield better bounds for the estimation of concurrence even in situations where the PPT criterion is optimal for the detection of entanglement. However, Eq. (8) should not be considered to render Eq. (2) obsolete but rather as a complement of it that can be used to refine the bounds that (2) provides when a suitable choice of LOOs is made. To determine what set of LOOs yields the best bound for a given state is left as an interesting open problem. We also think that this result helps to understand the relation between entanglement quantification and the LURs criterion. Like the results of [15] our bound can be attained by states having a particular optimal ensemble decomposition. This is the case of isotropic states [28] when $\left\{G_{i}^{A}\right\}=\left\{I / \sqrt{M}, w_{l} / \sqrt{2}, u_{j k} / \sqrt{2}, v_{j k} / \sqrt{2}\right\}$ and $\left\{G_{i}^{B}\right\}=\left\{-I / \sqrt{N},-w_{l} / \sqrt{2},-u_{j k} / \sqrt{2}, v_{j k} / \sqrt{2}\right\}$ are the chosen LOOs, and, hence, this explains the coincidence of concurrence and violation of LURs pointed out in [8].

We have also provided a similar lower bound on concurrence in terms of violations of the CM criterion for separability. Although this result is not as powerful as the one based on LURs, since it only seems to yield better bounds than what can be obtained using Eq. (2) in situations where the $\mathrm{CM}$ criterion has a stronger entanglement detection capability than both the PPT and CCNR criteria jointly, it provides a rigorous relation between concurrence and a correlation-based local unitary invariant measure, which is convenient from the experimental point of view as discussed in [17].

Finally, let us mention that the results presented here can be extended straightforwardly to yield lower bounds for the entanglement of formation by using the ideas of [30].

\section{ACKNOWLEDGMENTS}

The author acknowledges financial support by Universidad Carlos III de Madrid and Comunidad Autónoma de Madrid (project No. CCG06-UC3M/ESP-0690) and by Dirección General de Investigación (Ministerio de Edu- 
cación y Ciencia of Spain) under grant MTM2006-13000-

[1] M.A. Nielsen and I.L. Chuang, Quantum Computation and Quantum Information (Cambridge University Press, 2000).

[2] A. Peres, Phys. Rev. Lett. 77, 1413 (1996).

[3] M. Horodecki, P. Horodecki, and R. Horodecki, Phys. Lett. A 223, 1 (1996).

[4] O. Rudolph, arXiv:quant-ph/0202121 v1.

[5] K. Chen and L.-A. Wu, Quant. Inf. Comput. 3, 193 (2003).

[6] J.I. de Vicente, arXiv:quant-ph/0607195v3 [Quant. Inf. Comput. (to be published)].

[7] M. Lewenstein, B. Kraus, J.I. Cirac, and P. Horodecki, Phys. Rev. A 62, 052310 (2000).

[8] H.F. Hofmann and S. Takeuchi, Phys. Rev. A 68, 032103 (2003).

[9] O. Gühne, Phys. Rev. Lett. 92, 117903 (2004); V. Giovannetti, Phys. Rev. A 70, 012102 (2004); O. Gühne and M. Lewenstein, ibid. 70, 022316 (2004); J.I. de Vicente and J. Sánchez-Ruiz, ibid. 71, 052325 (2005).

[10] M.B. Plenio and S. Virmani, Quant. Inf. Comput. 7, 1 (2007).

[11] C.H. Bennett, D.P. DiVincenzo, J.A. Smolin, and W.K. Wootters, Phys. Rev. A 54, 3824 (1996).

[12] W.K. Wootters, Phys. Rev. Lett. 80, 2245 (1998).

[13] P. Rungta, V. Bužek, C.M. Caves, M. Hillery, and G.J. Milburn, Phys. Rev. A 64, 042315 (2001).

[14] A. Loziński, A. Buchleitner, K. Zyczkowski, and T. Wellens, Europhys. Lett. 62, 168 (2003); F. Mintert, M. Kuś, and A. Buchleitner, Phys. Rev. Lett. 92, 167902 (2004); F. Mintert and A. Buchleitner, arXiv:quant-ph/0605250v3 [Phys. Rev. Lett. (to be published)].

[15] K. Chen, S. Albeverio, and S.-M. Fei, Phys. Rev. Lett.
95, 040504 (2005).

[16] I. Ali Khan and J.C. Howell, Phys. Rev. A 70, 062320 (2004); S. Samuelsson and G. Björk, ibid. 73, 012319 (2006).

[17] C. Kothe and G. Björk, Phys. Rev. A 75, 012336 (2007).

[18] H.-P. Breuer, J. Phys. A: Math. Gen. 39, 11847 (2006).

[19] H.-P. Breuer, Phys. Rev. Lett. 97, 080501 (2006).

[20] J. Eisert, F.G.S.L. Brandão, and K.M.R. Audenaert, New J. Phys. 9, 46 (2007); O. Gühne, M. Reimpell, and R.F. Werner, Phys. Rev. Lett. 98, 110502 (2007).

[21] R.F. Werner, Phys. Rev. A 40, 4277 (1989).

[22] M. Horodecki, P. Horodecki, and R. Horodecki, Open Syst. Inf. Dyn. 13, 103 (2006).

[23] H.F. Hofmann, Phys. Rev. A 68, 034307 (2003).

[24] O. Gühne, M. Mechler, G. Tóth, and P. Adam, Phys. Rev. A 74, 010301(R) (2006).

[25] S. Yu and N.L. Liu, Phys. Rev. Lett. 95, 150504 (2005).

[26] C.H. Bennett, D.P. DiVincenzo, T. Mor, P.W. Shor, J.A. Smolin, and B.M. Terhal, Phys. Rev. Lett. 82, 5385 (1999).

[27] F.T. Hioe and J.H. Eberly, Phys. Rev. Lett. 47, 838 (1981).

[28] M. Horodecki and P. Horodecki, Phys. Rev. A 59, 4206 (1999).

[29] J.I. de Vicente, in preparation.

[30] K. Chen, S. Albeverio, and S.-M. Fei, Phys. Rev. Lett. 95, 210501 (2005).

[31] Except for the case of the negativity [G. Vidal and R.F. Werner, Phys. Rev. A 65, 032314 (2002)], which relies on the Peres-Horodecki criterion and therefore fails to quantify the entanglement of PPT entangled states. 